\title{
Critical phenomena in gravitational collapse of Husain-Martinez-Nunez scalar field
}

\author{
Xiaobao Wang ${ }^{1,2, a}$, Xiaoning $\mathbf{W u}^{3, \mathrm{~b}}$, Sijie Gao ${ }^{1, \mathrm{c}}$ \\ ${ }^{1}$ Department of Physics, Beijing Normal University, Beijing 100875, China \\ ${ }^{2}$ College of Science, Beijing Information Science and Technology University, Beijing 100192, China \\ ${ }^{3}$ Institute of Applied Mathematics, Academy of Mathematics and System Science, Chinese Academy of Sciences, P.O. Box 2734, Beijing 100080, \\ China
}

Received: 21 August 2019 / Accepted: 21 September 2019 / Published online: 8 October 2019

(C) The Author(s) 2019

\begin{abstract}
We construct analytical models to study the critical phenomena in gravitational collapse of the HusainMartinez-Nunez massless scalar field. We first use the cutand-paste technique to match the conformally flat solution ( $c=0$ ) onto an outgoing Vaidya solution. To guarantee the continuity of the metric and the extrinsic curvature, we prove that the two solutions must be joined at a null hypersurface and the metric function in Vaidya spacetime must satisfy certain constraints. We find that the mass of the black hole in the resulting spacetime takes the form $M \propto\left(p-p^{*}\right)^{\gamma}$, where the critical exponent $\gamma$ is equal to 0.5 . For the case $c \neq 0$, we show that the scalar field must be joined onto two pieces of Vaidya spacetimes to avoid a naked singularity. We also derive the power-law mass formula with $\gamma=0.5$. Compared with previous analytical models which were constructed from a different scalar field with continuous self-similarity, we obtain the same value of $\gamma$. However, we show that the solution with $c \neq 0$ is not self-similar. Therefore, we provide a rare example that a scalar field without self-similarity also possesses the features of critical collapse.
\end{abstract}

\section{Introduction}

Gravitational collapse is the main reason of various galactic structures and it remains one of the most interesting and fundamental problems in general relativity. The end state of gravitational collapse could be a black hole, naked singularity or flat spacetime. Since the pioneer work by Oppenheimer and Snyder [1], collapsing scenarios of different matter fields have been studied. Particularly, collapse of scalar

\footnotetext{
a e-mail: bao@mail.bnu.edu.cn

be-mail:wuxn@amss.ac.cn

ce-mail: sijie@bnu.edu.cn
}

field has attracted great attention in the past decades [2-7]. In a seminal work by Choptuik [8], some intriguing and universal properties concerning the formation of black holes from massless scalar fields were found. This is called the critical phenomenon. He found that, near the threshold, the black hole mass can always be expressed in the form of power-law:

$M \propto\left(p-p^{*}\right)^{\gamma}$,

for $p>p^{*}$, where $p$ is a parameter of the initial data to the threshold of black hole formation. Numerical simulations have shown that the critical exponent $\gamma$ is equal to 0.5 for solutions with continuous self-similarity (CSS) and $\gamma \approx 0.37$ for solutions with discrete self-similarity (DSS). Details about the critical phenomenon can be learned in $[9,10]$.

In addition to numerical calculation, analytical models were also built to explore the critical phenomena. Patrick R. Brady [11] studied an exact one parameter family of scalar field solutions which exhibits critical behaviours when black hole forms. Soda and Hirata [12] analytically studied the collapse of continuous self-similar scalar field in higher dimensional spacetimes and found a general formula for the critical exponents which agrees with the exponent $\gamma=0.5$ for $n=4$. Wang et al. [13] constructed an analytical model by pasting the BONT model (a massless scalar field) with the Vaidya model. They demonstrated that the black hole mass obeys the power law with $\gamma=0.5$. Wang et al. [14] also analytically studied the gravitational collapse of a massless scalar field with conformal flatness. They showed that the mass of the black hole without self-similarity turns on with finite nonzero values. Recent developments regarding the critical phenomenon can be found in Refs. [15-22].

Previously, the studies of critical phenomena concerns black holes in non-dynamical backgrounds. It would be interesting to investigate solutions of Einstein's equation in a cos- 
mological background. The McVittie spacetime [23] discovered in 1933 is regarded as the first analytical solution interpreted as describing a central object embedded in an FLRW universe. In this paper, we investigate the critical phenomena associated with an exact scalar field solution discovered by Husain-Martinez-Nunez (HMN) [24], which represents a black hole in an FLRW universe [25-27]. It was pointed out that the HMN solution brings in new phenomenology(Scurve) of apparent horizon [28]. Moreover, the conformally transformed HMN spacetime can be an inhomogeneous vacuum solution in Brans-Dick theory [29-31].

Since the HMN solution is not asymptotically flat, we need join it onto an exterior solution with asymptotic flatness. The Vaidya spacetime is a possible candidate because it describes a dynamic black hole and the free function $m(U)$ in the metric can be used to guarantee the continuity of the resulting spacetime. Following the treatment in [13], we match the HMN solution onto an outgoing Vaidya solution along a null hypersurface. Usually, the hypersurface connecting the two parts of the spacetime is a thin shell, i.e., the extrinsic curvature across the hypersurface is discontinuous. By applying the Darmois-Israel formula [33], one can find the relationship between the jump of the extrinsic curvature and the surface stress-energy tensor of the thin shell. However, by properly choosing the function $m(U)$ in the Vaidya metric, we find that the extrinsic metric can be continuous across the null surface. Therefore, no thin shell forms and the resulting spacetime can be at least $C^{1}$.

After the matching, we calculate the apparent horizon and define the black hole mass as the Komar mass at the intersection of the apparent horizon and the null hypersurface. There are two parameters, $a$ and $c$ in the HMN metric, whose values determine the staticity and homogeneity features of the solution. We first study the case $c=0$ where the HMN solution is conformally flat and has CSS. The mass of the black hole is found in the power-law form $M \propto \sqrt{-a}$ for $a<0$, which means $\gamma=0.5$. When $a=0$, the black hole disappears and the spacetime becomes Minkowski.

The case of $c \neq 0$ is more complicated. This solution has no self-similarity. We still find $M \propto \sqrt{-a}$ for $a<0$. Differing from the case of $c=0$, we show that the limiting spacetime $(a=0)$ possesses a naked singularity with nonzero ADM mass. Therefore, there is a mass gap between the black hole spacetime $(a<0)$ and the spacetime with naked singularity $a=0$.

The paper is organized as follows. In Sect. 2, we briefly introduce the Husain-Martinez-Nunez (HMN) scalar field solution. In Sect. 3, by using the cut-and-paste method, we match the conformally flat HMN solution $(c=0$ with CSS) onto an outgoing Vaidya spacetime at a null hypersurface. This matching guarantees the continuity of the metric and extrinsic curvature across the surface. Then, we use this analytical model to study the critical phenomenon and derive the mass formula. In Sect. 4, we join a general HMN $(a \neq 0, c \neq 0)$ with two outgoing Vaidya spacetimes. We show that the mass of the black hole approaches zero for $a<0$. We also find that the critical spacetime $(a=0)$ possesses a naked singularity with nonzero ADM mass. Concluding remarks are given in Sect. 5. In Appendix A, we show that the HMN solution and the Vaidya solution cannot be matched through a timelike hypersurface. In Appendix B, we prove that the HMN solution has CSS only when $a \neq 0$ and $c=0$.

\section{Husain-Martinez-Nunez (HMN) spacetime}

The Husain-Martinez-Nunez spacetime [24] satisfies the Einstein-scalar field equations

$$
\begin{aligned}
G_{a b} & =8 \pi T_{a b}, \\
T_{a b} & =\nabla_{a} \Phi \nabla_{b} \Phi-\frac{1}{2} g_{a b} g^{c d} \nabla_{c} \Phi \nabla_{d} \Phi .
\end{aligned}
$$

The spherically symmetric solution is given by ${ }^{1}$

$$
\begin{gathered}
d s^{2}=(a t+1)\left[-\left(1-\frac{2 c}{r}\right)^{\alpha} d t^{2}+\left(1-\frac{2 c}{r}\right)^{-\alpha} d r^{2}\right. \\
\left.+r^{2}\left(1-\frac{2 c}{r}\right)^{1-\alpha} d \Omega^{2}\right] \\
\Phi(r, t)= \pm \frac{1}{4 \pi} \ln \left[\left(1-\frac{2 c}{r}\right)^{\frac{\alpha}{\sqrt{3}}}(a t+1)^{\sqrt{3}}\right]
\end{gathered}
$$

where $\alpha= \pm \frac{\sqrt{3}}{2}$. We shall focus on the case $-\infty \leq t \leq-\frac{1}{a}$ and $a<0$ because it corresponds to a black hole solution. In this paper, we only study the case $\alpha=-\frac{\sqrt{3}}{2}$ for simplicity. The results remain true for $\alpha=\frac{\sqrt{3}}{2}$.

From the Ricci scalar

$$
\begin{gathered}
\mathcal{R}=\frac{12 c a^{2}(r-c)-3 a^{2} r^{2}}{2 r^{2}(a t+1)^{3}}\left(1-\frac{2 c}{r}\right)^{-2-\alpha} \\
+\frac{2 c^{2}\left(1-\alpha^{2}\right)}{(a t+1) r^{4}}\left(1-\frac{2 c}{r}\right)^{-2+\alpha}
\end{gathered}
$$

we see that the curvature singularities are located at $r=2 c$ (timelike singularity) and $t=-1 / a$ (spacelike singularity).

To find the apparent horizon, we first write down the tangents to the null geodesics in the the radial direction:

$$
\begin{aligned}
\frac{\partial}{\partial \lambda^{+}} & =\frac{1}{(a t+1)}\left(1-\frac{2 c}{r}\right)^{-\alpha} \frac{\partial}{\partial t}+\frac{1}{(a t+1)} \frac{\partial}{\partial r}, \\
\frac{\partial}{\partial \lambda^{-}} & =\frac{1}{(a t+1)}\left(1-\frac{2 c}{r}\right)^{-\alpha} \frac{\partial}{\partial t}-\frac{1}{(a t+1)} \frac{\partial}{\partial r},
\end{aligned}
$$

\footnotetext{
1 Without loss of generality, we have set $b=1$ in the original metric appearing in [24].
} 
where $\lambda_{ \pm}$represent affine parameters. The corresponding expansions are given by

$\Theta_{+}=\frac{1}{\sqrt{h}} \frac{\partial \sqrt{h}}{\partial \lambda^{+}}$,

$\Theta_{-}=\frac{1}{\sqrt{h}} \frac{\partial \sqrt{h}}{\partial \lambda^{-}}$

where $\sqrt{h}=R^{2} \sin \theta=(a t+1) r^{2}\left(1-\frac{2 c}{r}\right)^{1-\alpha} \sin \theta$. Then straightforward calculation yields

$$
\begin{aligned}
& \Theta_{+}=\left\{a r^{2}\left(1-\frac{2 c}{r}\right)^{-\alpha}+\left[2 r-\frac{2 c r(\alpha-1)}{r-2 c}\right](a t+1)\right\} \\
& \Theta_{-}=\left\{a r^{2}\left(1-\frac{2 c}{r}\right)^{-\alpha}-\left[2 r-\frac{2 c r(\alpha-1)}{r-2 c}\right](a t+1)\right\} \\
& r^{-2}(a t+1)^{-2} .
\end{aligned}
$$

The apparent horizon satisfies $\Theta_{+}=0, \Theta_{-}<0$, which is located at

$\frac{a}{a t_{A H}+1}=-\frac{2}{r_{A H}^{2}}\left[r_{A H}-c(1+\alpha)\right]\left(1-\frac{2 c}{r_{A H}}\right)^{\alpha-1}$.

The Misner-Sharp mass [34] is defined by

$M=\frac{R}{2}\left(1-g^{a b} \nabla_{a} R \nabla_{b} R\right)$

where $R$ denotes the areal radius. From [28], we know that in spherically symmetric spacetimes, the apparent horizon satisfies

$g^{a b} \nabla_{a} R \nabla_{b} R=0$.

Therefore, on the apparent horizon, the Misner-sharp mass becomes

$M=\frac{R}{2}$.

\section{Critical behaviour of HMN scalar field with conformal flatness $(c \neq 0)$}

To study the critical phenomena of HMN massless scalar field, we start with the simple case $c=0$, where the spacetime is conformally flat [24]. First, we need to join the HMN solution onto an outgoing Vaidya solution such that the resulting spacetime is asymptotically flat. In Appendix A, we have shown that the two spacetimes cannot be matched at a timelike boundary. In the following subsection, we shall replace the timelike hypersurface with a null hypersurface.
3.1 Matching at a null hypersurface

Matching the two solutions at a null hypersurface is more complicated than at a timelike hypersurface. We shall follow the method in [13] and [32]. First we use the coordinate transformation $v=t+r$ to replace the coordinate $r$ in Eq. (4) and obtain the metric in the interior

$d s^{2}=-[a(v-r)+1] d v(d v-2 d r)+R^{2}\left(d \theta^{2}+\sin ^{2} \theta d \phi^{2}\right)$,

where

$R^{2}=[a(v-r)+1] r^{2}$.

Let $\Sigma$ be the null hypersurface $v=v_{0}$ (see Fig. 1). We shall use "-" to label the inner HMN spacetime and "+" to label the exterior Vaidya spacetime. The normal to $\Sigma$ is

$n_{a}^{-}=s^{-1} d v_{a}$,

where $s$ is an arbitrary negative function such that $n_{-}^{a}$ is a future directed vector. We can introduce a transverse null vector $N_{a}$ by requiring

$n_{a} N^{a}=-1$

$N^{a} N_{a}=0$.

It is easy to show that

$N_{a}^{-}=s[a(v-r)+1]\left(\frac{1}{2} d v_{a}-d r_{a}\right)$.

Now we choose $s=-\frac{1}{a(v-r)+1} \frac{\partial R}{\partial r}$. Let $\xi^{i}=\{R, \theta, \phi\}$ be the intrinsic coordinates on $\Sigma$. Then $\Sigma$ is determined by

$v=v_{0}, \quad r=r(R), \quad \theta=\theta, \quad \phi=\phi$

where $r(R)$ is given by Eq. (18) with $v=v_{0}$. We define $e_{(a)}^{-\mu} \equiv \frac{\partial x_{-}^{\mu}}{\partial \xi^{a}}$ as given in the [13]. Thus

$$
\begin{aligned}
e_{(1)}^{-r} & =\frac{2 r R}{2 R^{2}-a r^{3}}, \\
e_{(2)}^{-\theta} & =1, \\
e_{(3)}^{-\phi} & =1 .
\end{aligned}
$$

Similarly to Eq. (75), the transverse extrinsic curvature for the null surface is given by [32]

$k_{i j}=-N_{\mu} \frac{\partial^{2} x^{\mu}}{\partial \xi^{i} \xi^{j}}-N_{\nu} \Gamma_{\mu \rho}^{\nu} \frac{\partial x^{\mu}}{\partial \xi^{i}} \frac{\partial x^{\rho}}{\partial \xi^{j}}$ 


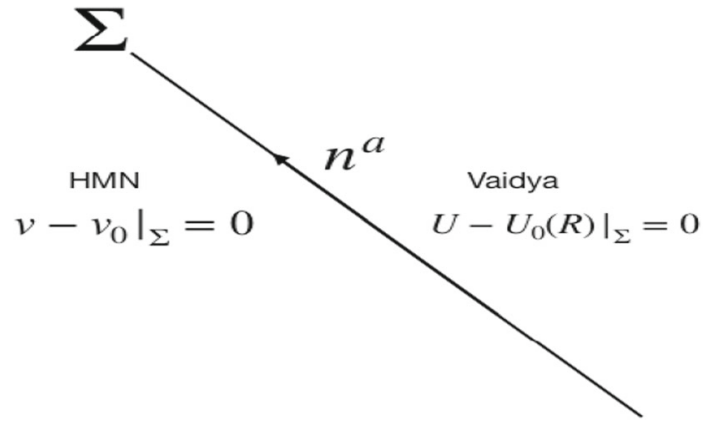

Fig. 1 Null hypersurface $\Sigma$

By straightforward calculation, we find

$$
\begin{aligned}
k_{a b}^{-}= & \frac{-3 a^{2} r}{\left(3 a r-2 a v_{0}-2\right)^{2} \sqrt{a\left(v_{0}-r\right)+1}} d R_{a} d R_{b} \\
& +\frac{r\left[a\left(r-2 v_{0}\right)-2\right]\left(3 a r-2 a v_{0}-2\right)}{8\left[a\left(v_{0}-r\right)+1\right]^{\frac{3}{2}}} \\
& \times\left(d \theta_{a} d \theta_{b}+\sin \theta^{2} d \phi_{a} d \phi_{b}\right) .
\end{aligned}
$$

On the other hand, we need to join the HMN solution to the exterior Vaidya spacetime (70) at $\Sigma$, as shown in Fig. 1. Assume that the null surface $\Sigma$ can be described by $U=$ $U_{0}(R)$ from the Vaidya solution. It follows from (70) that

$\frac{d U_{0}}{d R}=-\frac{2 R}{R-2 m\left[U_{0}(R)\right]}$
The continuity condition on $\Sigma$ requires [32]

$\left.N_{\mu}^{+} e_{(i)}^{+\mu}\right|_{v=v_{0}}=\left.N_{\mu}^{-} e_{(i)}^{-\mu}\right|_{v=v_{0}}$

This also guarantees that the normal vectors $n^{a}$ defined on both sides are the the same. According to Eqs. (22), (24), (29) and (31), we find that the nontrivial equations in Eq. (32) are

$\left.N_{\mu}^{-} e_{(1)}^{-\mu}\right|_{v=v_{0}}=N_{r}^{-} e_{(1)}^{-r}=1$,

$\left.N_{\mu}^{+} e_{(1)}^{+\mu}\right|_{v=v_{0}}=N_{U}^{+} e_{(1)}^{+U}=-\beta$.

Hence, we get $\beta=-1$.

Now we can calculate the corresponding transverse extrinsic curvature from Eqs. (25) and obtain

$$
\begin{aligned}
k_{a b}^{+}= & \frac{-2 m^{\prime}(r) r^{\prime}(R)}{\sqrt{a\left(v_{0}-r\right)+1} r-2 m(r)} d R_{a} d R_{b} \\
& +\left(\frac{R}{2}-m\left[U_{0}(R)\right]\right)\left(d \theta_{a} d \theta_{b}+\sin ^{2} \theta d \phi_{a} d \phi_{b}\right) .
\end{aligned}
$$

Since $k_{a b}^{+}=k_{a b}^{-}$, Eqs. (26) and (35) give rise to

$\left.k_{R R}^{+}\right|_{\Sigma}=\left.k_{R R}^{-}\right|_{\Sigma}$

By integration, we obtain $m(r)$ as

$m(r)=\frac{-8+a^{3}\left(-27 r^{3}+12 r v_{0}^{2}-8 v_{0}^{3}\right)+12 a\left[r-2\left(v_{0}+18 c_{1}\right)\right]+24 a^{2}\left[-v\left(v_{0}+18 c_{1}\right)+r\left(v_{0}+27 c_{1}\right)\right]}{-216 a\left[1+a\left(v_{0}-r\right)\right]^{\frac{3}{2}}}$,

The spacetime coordinates $\left\{x_{+}^{\mu}\right\}$ can be expressed as functions of $\xi^{i}$ :

$U=U_{0}(R), \quad R=R, \quad \theta=\theta, \quad \phi=\phi$.

Define $e_{(i)}^{+\mu} \equiv \frac{\partial x_{+}^{\mu}}{\partial \xi^{i}}$ and it is easy to find

$e_{(1)}^{+U}=-\frac{2}{f_{+}}, \quad e_{(1)}^{+R}=e_{(2)}^{+\theta}=e_{(3)}^{+\phi}=1$.

The normal to $\Sigma$ is given by

$n_{a}^{+}=\beta^{-1} \nabla_{a}\left(U-U_{0}(R)\right)=\beta^{-1}\left(d U_{a}+\frac{2}{f_{+}} d R_{a}\right)$.

where $\beta$ is a negative function which will be determined later. Then the transverse null vector $N^{a}$ satisfying Eq. (20) is

$N_{a}^{+}=\frac{\beta f_{+}}{2} d U_{a}$ where $c_{1}$ is an integral constant. Using $\left.k_{\theta \theta}^{+}\right|_{\Sigma}=\left.k_{\theta \theta}^{-}\right|_{\Sigma}$, we can fix $c_{1}$ :

$c_{1}=\frac{-\left(a v_{0}+1\right)^{2}}{54 a}$.

Consequently,

$m(r)=\frac{a^{2} r^{3}}{8\left[a\left(v_{0}-r\right)+1\right]^{3 / 2}}$.

Note that

$\left.m(r)\right|_{\Sigma}=m\left(\left.U(R(r))\right|_{\Sigma}\right.$

So, Eq. (39) together with Eq. (27) specifies a unique metric function $m(U)$ in the Vaidya solution. Therefore, we have matched the conformally flat spacetime with Vaidya spacetime at the null hypersurface. 


\subsection{Mass of the black hole}

From Eq. (14), one can calculate the Misner-Sharp mass for HMN spacetime described by metric (69) and obtain

$M=\frac{r^{3} a^{2}}{8(a t+1)^{\frac{3}{2}}}$.

Therefore, $m(r)$ in Eq. (39) is just the Misner-Sharp mass at $v=v_{0}$. The apparent horizon determined by Eq. (13) takes the simple form for $c=0$ :

$\frac{a}{a t_{A H}+1}=-\frac{2}{r_{A H}}$.

Note that the null surface is determined by

$v_{0}=t+r$.

Equations (42) and (43) immediately gives the coordinates at the intersection of $\Sigma$ and the apparent horizon:

$r_{i}=2 v_{0}+\frac{2}{a}$,

$t_{i}=-v_{0}-\frac{2}{a}$.

Since $r>0$ and $a<0$, from Eq. (44), we see that the existence of the intersection requires

$v_{0}>|a|^{-1}$

Therefore, the Misner-Sharp mass at the intersection is

$M_{i}=\sqrt{-a}\left(v_{0}+\frac{1}{a}\right)^{\frac{3}{2}}$.

The event horizon coincides with the apparent horizon in the outgoing Vaidya spacetime as shown in Fig. 2. It is also known that the mass function $m$ in the Vaidya metric is constant alone the event horizon [35]. Thus, it is natural to take the mass in Eq. (47) to be the mass of the black hole.

To investigate the critical behavior as $a \rightarrow 0$, we impose the condition that $r_{i}$ in Eq. (45) does not change with $a$. This means that $v_{0}$ must take the form

$v_{0}=V-\frac{1}{a}$,

where $V$ is a positive constant independent of $a$. Thus, Eq. (47) gives the mass of black hole:

$M_{b h}=\sqrt{-a} V^{\frac{3}{2}}$.

Equation (49) shows that the mass of black hole can be put in the form of Eq. (1) and the scaling exponent is $\gamma=0.5$.

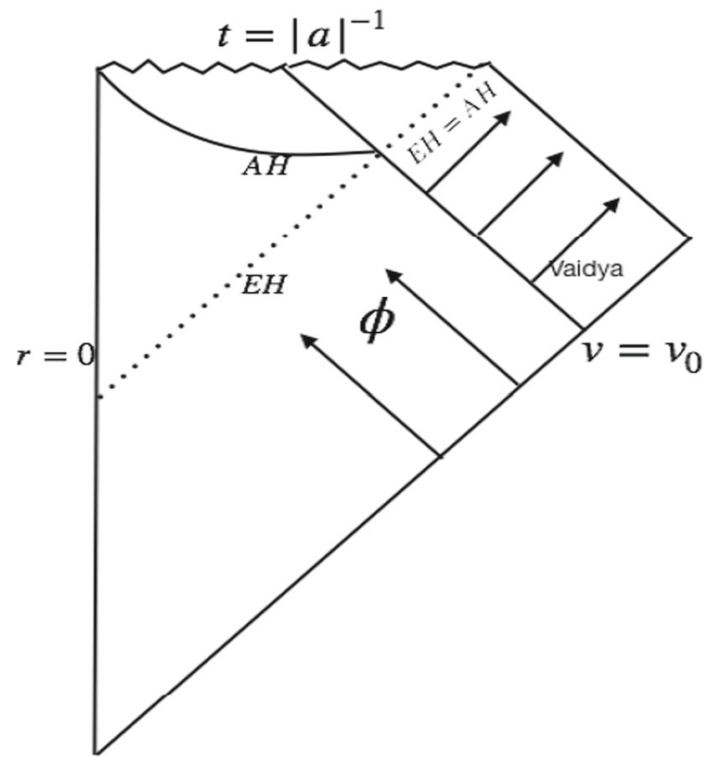

Fig. 2 Penrose diagram for the HMN spacetime $(a \neq 0, c=0)$ matching with an outging Vaidya spacetime at $v=v_{0}$. The singularity is located at $t=-a^{-1}$

Obviously, as $a$ approaches zero, the mass of the black hole vanishes and the spacetime becomes Minkowski.

\section{Collapse of the general HMN scalar field}

In this section, we shall investigate the gravitational collapse associated with a general HMN scalar field $(a \neq 0, c \neq 0)$.

\subsection{Matching to an outgoing Vaidya solution at a null hypersurface}

Under the coordinate transformation

$v=t+h(r)$

Equation (4) can be rewritten in the form

$$
\begin{aligned}
& d s^{2}=-[a(v-h(r))+1]\left(1-\frac{2 c}{r}\right)^{\alpha}\left(d v^{2}-2 h^{\prime}(r) d v d r\right) \\
& +a[(v-h(r))+1]\left(1-\frac{2 c}{r}\right)^{1-\alpha} r^{2} d \Omega^{2}
\end{aligned}
$$

Here, the function $h(r)$ satisfies

$h^{\prime}(r)=\left(1-\frac{2 c}{r}\right)^{-\alpha}$.

The areal radius $R$ takes the form

$R=\sqrt{a(v-h(r))+1}\left(1-\frac{2 c}{r}\right)^{\frac{1-\alpha}{2}} r$. 


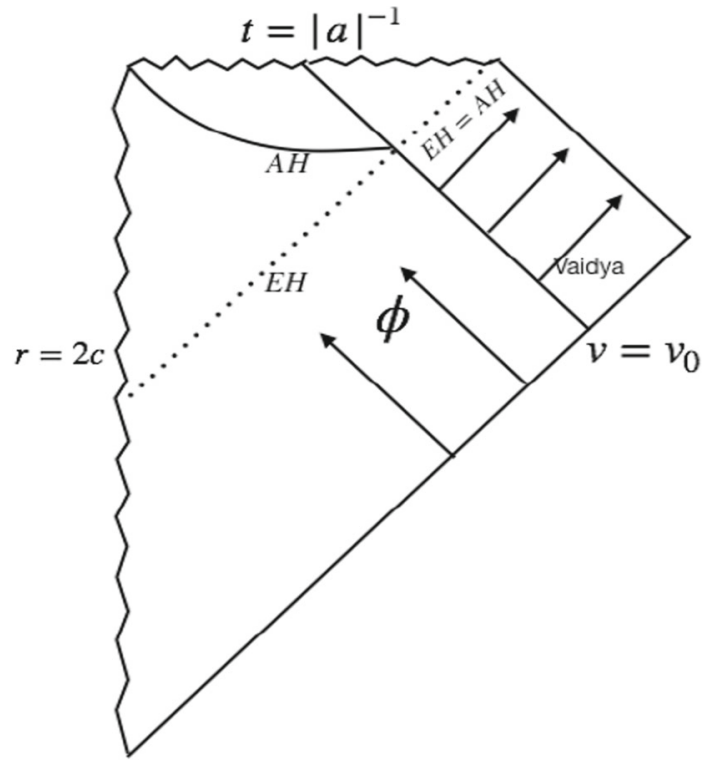

Fig. 3 Penrose diagram for the HMN solution $(a \neq 0, c \neq 0, \alpha=$ $-\frac{\sqrt{3}}{2}$ ) matching to an outging Vaidya solution. There are two singularities at $t=\left|\frac{1}{a}\right|$ and $r=2 c$, where $r=2 c$ is a naked singularity

Similarly to Sect. 3, we match the solution onto an outgoing Vaidya solution at the null hypersurface $v=v_{0}$ (see Fig. 3). Substitution of $v=v_{0}$ into Eq. (53) yields the function $r=r(R)$. By the method in Sect. 3.1, the extrinsic curvature can be calculated as
It is easy to see that $k_{a b}^{+}$takes the same form as in Eq. (35). Then $k_{a b}^{-}=k_{a b}^{+}$yields

$$
\begin{aligned}
m(r)= & \frac{1}{2}(1-2 c / r)^{(2-\sqrt{3}) / 4} r \sqrt{a\left(v_{0}-h(r)\right)+1} \\
& +\frac{1}{4\left(a\left(v_{0}-h(r)\right)+1\right) r^{\prime}(R)}((2+\sqrt{3}) c-2 r \\
& +4 a c r(1-2 c / r)^{-\frac{\sqrt{3}}{2}}-2 a(1-2 c / r)^{-\frac{\sqrt{3}}{2}} r^{2} \\
& +((2+\sqrt{3}) c-2 r) a v_{0} \\
& \left.-a((2+\sqrt{3}) c-2 r) h(r)+\operatorname{ar}(-2 c+r) h^{\prime}(r)\right) .
\end{aligned}
$$

By our construction, the metric of the resulting spacetime is continuous and the extrinsic curvature of the null hypersurface is also continuous. Therefore, we have shown that the general HMN spacetime ( $a \neq 0, c \neq 0)$ and Vaidya spacetime can be matched at a null hypersurface as showed in Fig. 3.

\subsection{Mass of the black hole}

By the argument in Sect. 3.2, one can show that $m(r)$ in Eq. (57) is exactly the Misner-sharp mass for the metric in Eq. (51). Therefore, from Eqs. (13), (16) and (53), we obtain the mass on the apparent horizon:

$m_{A H}(r)=\frac{R_{A H}(r)}{2}=\sqrt{-a} r^{2}[8 r-8 c(1+\alpha)]^{-\frac{1}{2}}\left(1-\frac{2 c}{r}\right)^{-\alpha+1}$.

$$
\begin{aligned}
k_{a b}^{-}= & \left(\frac{a h^{\prime}(r) r^{\prime}(R)}{1+a\left(v_{0}-h(r)\right)}-\frac{r^{\prime \prime}(R)}{r^{\prime}(R)}\right) d R_{a} d R_{b}-\left[\frac{(2+\sqrt{3}) c-2 r+4 a c(1-2 c / r)^{-\frac{\sqrt{3}}{2}} r-2 a(1-2 c / r)^{-\frac{\sqrt{3}}{2}} r^{2}}{4\left[1+a\left(v_{0}-h(r)\right)\right] r^{\prime}(R)}\right. \\
& \left.+\frac{((2+\sqrt{3}) c-2 r) a h(r)+2 a r v_{0}+a r(2 c-r) h^{\prime}(r)-(2+\sqrt{3}) a c v_{0}}{4\left[1+a\left(v_{0}-h(r)\right)\right] r^{\prime}(R)}\right]\left(d \theta_{a} d \theta_{b}+\sin ^{2} \theta d \phi_{a} d \phi_{b}\right) .
\end{aligned}
$$

where

$$
\begin{aligned}
r^{\prime}(R)= & -\frac{2\left(1-\frac{2 c}{r}\right)^{\frac{\alpha-1}{2}}(r-2 c) \sqrt{a\left[v_{0}-h(r)\right]}}{((2+\sqrt{3}) c-2 r)\left(1+a v_{0}\right)-a((2+\sqrt{3}) c-2 r) h(r)+a r(-2 c+r) h^{\prime}(r)}, \\
r^{\prime \prime}(R)= & -\left[((2+\sqrt{3}) c-2 r)\left(1+a v_{0}\right)-a((2+\sqrt{3}) c-2 r) h(r)+a r(-2 c+r) h^{\prime}(r)^{3}\right]^{-3} \\
& +\left\{2 ( 1 - 2 c / r ) ^ { \frac { \sqrt { 3 } } { 2 } } \left[a^{2} c^{2} h(r)^{2}+2 a((2+\sqrt{3}) c-2 r)(2 c-r) r\left(1+a v_{0}\right) h^{\prime}(r)\right.\right. \\
& +a^{2} r^{2}(-2 c+r)^{2} h^{\prime}(r)^{2}-2 a h(r)\left(c^{2}\left(1+a v_{0}\right)+a((2+\sqrt{3}) c-2 r)(2 c-r) r h^{\prime}(r)\right. \\
& \left.\left.\left.+a r^{2}(-2 c+r)^{2} h^{\prime \prime}(r)\right)+\left(1+a v_{0}\right)\left(c^{2}\left(1+a v_{0}\right)+2 a r^{2}(-2 c+r)^{2} h^{\prime \prime}(r)\right)\right]\right\} .
\end{aligned}
$$


Let $v_{0}$ be a constant and

$v_{0}=t+h(r)$

determines a null hypersurface which serves as the boundary of the two spacetimes. From Eqs. (13) and (58), we can get the coordinates $\left(t_{i}, r_{i}\right)$ at the intersection of the apparent horizon and the null hypersurface $v=v_{0}$, which satisfy

$v_{0}+\frac{1}{a}=\frac{\left(1-2 c / r_{i}\right)^{\frac{\sqrt{3}}{2}}\left(2 c-r_{i}\right) r_{i}+\left[(-2+\sqrt{3}) c+2 r_{i}\right] h\left(r_{i}\right)}{(-2+\sqrt{3}) c+2 r_{i}}$

$a t_{i}+1=\frac{a\left(1-2 c / r_{i}\right)^{\frac{\sqrt{3}}{2}} r_{i}\left(2 c-r_{i}\right)}{-2 c+\sqrt{3} c+2 r_{i}}>0$.

Similarly, we choose the null hypersurface which intersects with the apparent horizon at a fixed radius $r_{i}=r_{0}$, which is independent of $a$. Again, we take the Misner-Sharp mass at the intersection as the black hole mass. Then, Eq. (57) gives the mass of the black hole

$M_{b h}\left(r_{0}\right)=\sqrt{-a} f\left(r_{0}, c\right)$,

where

$f\left(r_{0}, c\right)=r_{0}^{2}\left[8 r_{0}-8 c(1+\alpha)\right]^{-\frac{1}{2}}\left(1-\frac{2 c}{r_{0}}\right)^{-\alpha+1}$.

Equation (61) shows clearly that the black hole mass satisfies the power law with $\gamma=0.5$.

However, the spacetime possesses a naked singularity $r=2 c$ (see Fig. 3), in violation of the cosmic censorship conjecture. To remove the naked singularity, we join another outgoing Vaidya spacetime at $v=v_{1}\left(v_{1}<v_{0}\right)$, as shown in Fig. 4. In the new spacetime, the HMN solution is configured in between two Vaidya solutions and no naked singularity exists.

When we study the relation between the mass and the parameter $a$, we treat $c$ as a constant. We see that $M_{b h} \rightarrow 0$ as $a \rightarrow 0$. When $a=0$, there is no black hole but a naked singularity as discussed in Sect. 4.3.

\section{3 "Critical spacetime": $a=0$ and $c \neq 0$}

Now we study the critical HMN spacetime. For $a=0$, Eq. (4) becomes

$$
\begin{aligned}
d s^{2}= & -\left(1-\frac{2 c}{r}\right)^{\alpha} d t^{2}+\left(1-\frac{2 c}{r}\right)^{-\alpha} d r^{2} \\
& +r^{2}\left(1-\frac{2 c}{r}\right)^{1-\alpha} d \Omega^{2},
\end{aligned}
$$

To calculate the apparent horizon of the spacetime, we first choose two families of radial null vector fields

$$
\frac{\partial}{\partial \lambda^{+}}=\left(1-\frac{2 c}{r}\right)^{-\alpha} \frac{\partial}{\partial t}+\frac{\partial}{\partial r}
$$

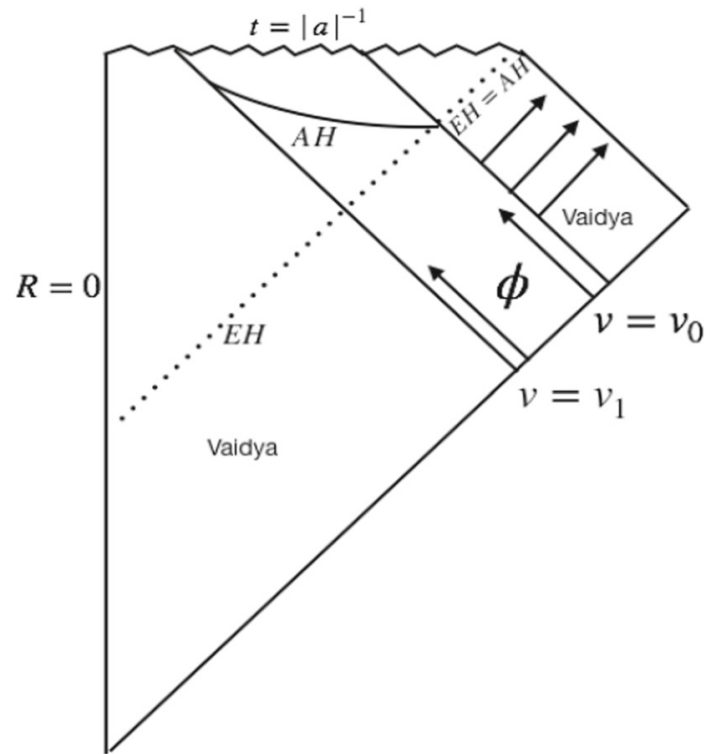

Fig. 4 Penrose diagram for the HMN scalar field $(a \neq 0, c \neq 0)$ matching with two outgoing Vaidya spacetimes. We see that the naked singularity in Fig. 3 has been replaced by the Vaidya spacetime

$\frac{\partial}{\partial \lambda^{-}}=\left(1-\frac{2 c}{r}\right)^{-\alpha} \frac{\partial}{\partial t}-\frac{\partial}{\partial r}$.

where $\lambda^{ \pm}$is the affine parameter of the null geodesic.

According to Eq. (11)

$\Theta_{+}=-\frac{2(c-r+c \alpha)}{r(-2 c+r)}$,

$\Theta_{-}=\frac{2(c-r+c \alpha)}{r(-2 c+r)}$.

Therefore, the spacetime has no apparent horizon when $r>$ 2c. According to Eq. (6), the spacetime possesses a naked singularity at $r=2 c$ (see Fig. 5).

Since the spacetime is asymptotically flat, we calculate the ADM mass and find

$M=c \alpha$.

When $a \neq 0$, the mass of the black hole is given by Eq. (61), which shows clearly that $M \rightarrow 0$ as $a \rightarrow 0$. However, when $a=0$, as we just discussed, the spacetime is not Minkowski and its ADM mass is nonzero. Therefore, there exists a mass gap between the black hole solution $(a \neq 0)$ and its limiting spacetime $(a=0)$.

\section{Conclusion}

In this paper, we have used the "cut and paste" method to construct analytical models and studied the critical phenomena of the HMN scalar filed. We have shown that the HMN solution with conformal flatness $(c=0)$ can be matched with 


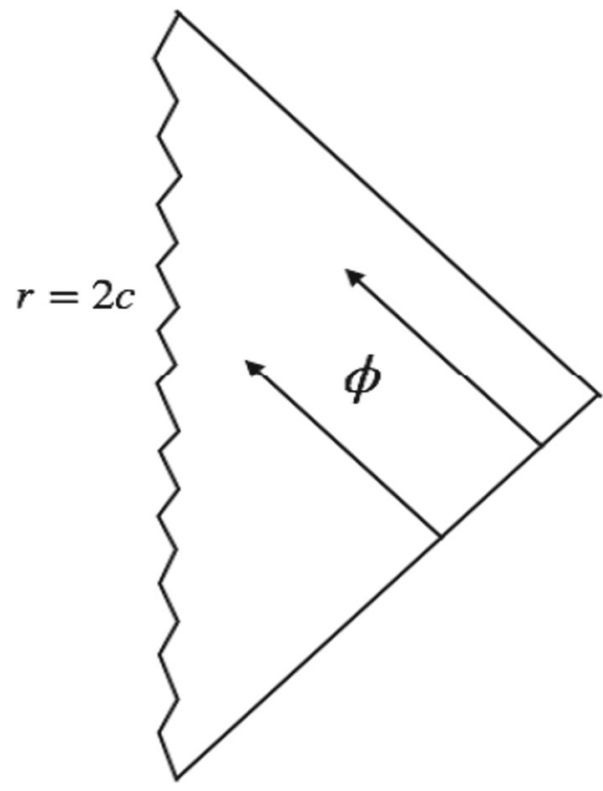

Fig. 5 Penrose diagram for the $\operatorname{HMN}$ spacetime $(a=0, c \neq 0)$. There is a naked singularity at $r=2 c$

the Vaidya solution along a null hypersurface, but not a timelike hypersurface. We have derived the differential equation which specifies the metric function in the Vaidya solution. For $c \neq 0$, we have joined the scalar field onto two patches of Vaidya spacetimes to avoid the naked singularity.

We have studied the gravitational collapse for the HMN scalar field and shown that black hole mass satisfies the power law with $\gamma=0.5$. This is consistent with previous results in the literature. When $c \neq 0$, the HMN spacetime has no CSS and the black hole also turns on at infinitely small mass. The result is different from the model in [14], which shows that the formation of black holes may turn on at finite mass when the gravitational collapse has no self-similarity. On the other hand, the mass gap exists between the black hole and the naked singularity during the gravitational collapse of HMN scalar field when $c \neq 0$ as discussed in Sect. 4.

Previous studies on the critical phenomena are associated black holes in static backgrounds. The HMN black hole is embedded in a cosmological background, which is dynamical. Many properties of black holes could change due to the dynamical background. This is why the model we constructed is special and gives some different results. Our work suggests that critical collapse can be studied from analytical models which are constructed by known solutions. More models should be investigated in the future in order to test universal features in gravitational collapse.

Acknowledgements This research was supported by NSFC Grants No. 11775022,11575286 and 11731001.
Data Availability Statement This manuscript has no associated data or the data will not be deposited. [Authors' comment: The paper is based on analytical solutions and no numerical data is involved.]

Open Access This article is distributed under the terms of the Creative Commons Attribution 4.0 International License (http://creativecomm ons.org/licenses/by/4.0/), which permits unrestricted use, distribution, and reproduction in any medium, provided you give appropriate credit to the original author(s) and the source, provide a link to the Creative Commons license, and indicate if changes were made.

Funded by $\mathrm{SCOAP}^{3}$.

\section{A Matching the HMN and Vaidya solutions at a timelike boundary}

In this appendix, we assume that the boundary connecting the two solutions is a timelike hypersurface. We shall use "-" to label the inner HMN spacetime and "+" to label the exterior Vaidya spacetime (see Fig. 6).

Setting $c=0$ in Eq. (4), the interior spacetime is described by the metric

$d s_{-}^{2}=(1+a t)\left[-d t^{2}+d r^{2}+r^{2}\left(d \theta^{2}+\sin \theta^{2} d \phi^{2}\right)\right]$.

The exterior spacetime is described by the outgoing Vaidya metric

$d s_{+}^{2}=-f_{+} d U^{2}-2 d U d R+R^{2} d \Omega^{2}$,

where $f_{+}=1-\frac{2 m(U)}{R}$. We choose $\xi^{i}=\{\lambda, \theta, \phi\}$ as the intrinsic coordinates on the hypersurface and denote $x^{\mu}=$ $(t, r, \theta, \phi)$. Then $\Sigma$ is determined by functions $\left\{x^{\mu}\left(\xi^{i}\right)\right\}$, i.e., $\{t(\lambda), r(\lambda)\}$ from the interior and $\{U(\lambda), R(\lambda)\}$ from the exterior. The induced metric on $\Sigma$ is given by

$\left.d s_{-}^{2}\right|_{\Sigma}=(a t+1)\left[\left(-\dot{t}^{2}+\dot{r}^{2}\right) d \lambda^{2}+r^{2}\left(d \theta^{2}+\sin \theta^{2} d \phi^{2}\right)\right]$,

$\left.d s_{+}^{2}\right|_{\Sigma}=-\left(f_{+} \dot{U}+2 \dot{R}\right) \dot{U} d \lambda^{2}+R^{2} d \Omega^{2}$.

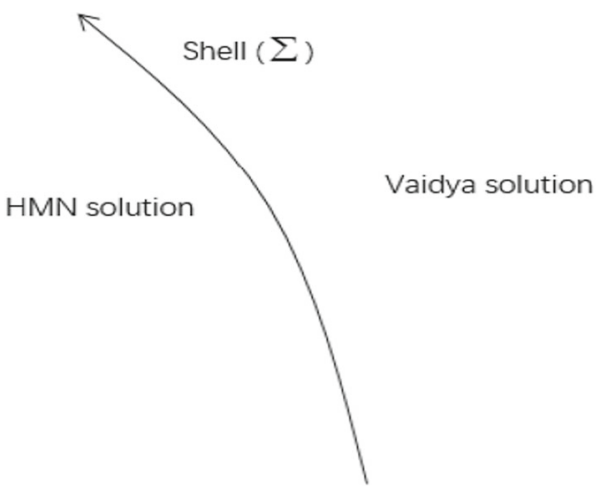

Fig. 6 The thin shell represented by a timelike hypersurface 
Here "." represents the derivative with respect to $\lambda$.

We use the Darmois junction conditions to match the two solutions across $\Sigma$ :

$\left.d s_{-}^{2}\right|_{\Sigma}=\left.d s_{+}^{2}\right|_{\Sigma}$,
$\left.k_{a b}^{-}\right|_{\Sigma}=\left.k_{a b}^{+}\right|_{\Sigma}$,

where $k_{a b}$ is the extrinsic curvature of $\Sigma$. The components of $k_{a b}$ can be calculated from

$k_{i j}=-n_{\mu} \frac{\partial^{2} x^{\mu}}{\partial \xi^{i} \xi^{j}}-n_{\nu} \Gamma_{\mu \rho}^{\nu} \frac{\partial x^{\mu}}{\partial \xi^{i}} \frac{\partial x^{\rho}}{\partial \xi^{j}}$

where $n_{a}$ is the spacelike normal to $\Sigma$. Computing $k_{i j}$ from the interior and exterior, respectively, we obtain the nonvanishing components:

$$
\begin{aligned}
k_{\lambda \lambda}^{-}= & \frac{a \dot{r}\left(\dot{r}^{2}-\dot{t}^{2}\right)-2(a t+1)(\ddot{t} \ddot{r}-\dot{r} \ddot{t})}{2 \sqrt{(a t+1)\left(\dot{t}^{2}-\dot{r}^{2}\right)}}, \\
k_{\lambda \lambda}^{+}= & \frac{\dot{U}}{\left(f_{+} \dot{U}^{2}+2 \dot{R} \dot{U}\right)^{\frac{1}{2}}}\left[-\frac{\dot{U}^{2}}{R}\left(\frac{m f_{+}}{R}-\frac{d m}{d U}\right)\right. \\
& \left.+\dot{R}\left(\frac{\dot{U}}{\dot{U}}-3 \frac{\dot{U} m}{R^{2}}\right)-\ddot{R}\right], \\
k_{\theta \theta}^{-}= & \frac{1}{\sin ^{2} \theta k_{\phi \phi}^{\mathcal{I}}}=\frac{a r^{2} \dot{r}+2(a t+1) \dot{t} r}{2 \sqrt{(a t+1)\left(\dot{t}^{2}-\dot{r}^{2}\right)}}, \\
k_{\theta \theta}^{+}= & \frac{1}{\sin ^{2} \theta k_{\phi \phi}^{\mathcal{E}}}=\frac{R}{\left(f_{+} \dot{U}^{2}+2 \dot{R} \dot{U}\right)^{\frac{1}{2}}}\left(\dot{R}+f_{+} \dot{U}\right) .
\end{aligned}
$$

Substituting Eqs. (71) and (72) into Eq. (73), we have

$$
\begin{aligned}
R & =\sqrt{a t+1} r, \\
(a t+1)\left(\dot{t}^{2}-\dot{r}^{2}\right) & =\left(f_{+} \dot{U}+2 \dot{R}\right) \dot{U} .
\end{aligned}
$$

Substituting Eqs. (78) and (79) into Eq. (74), with the help of Eq. (81), we obtain

$f_{+} \dot{U}+\dot{R}=\frac{a r \dot{r}+2(a t+1) \dot{t}}{2 \sqrt{a t+1}}$.

Equation (80) yields

$\dot{R}=\frac{a r \dot{t}}{2 \sqrt{(a t+1)}}+\sqrt{a t+1} \dot{r}$

Therefore, one can solve Eqs. (81)-(83) and obtain

$$
\begin{aligned}
\dot{U} & =\frac{2(a t+1)^{\frac{3}{2}}(\dot{t}-\dot{r})}{a r+2(a t+1)}, \\
f_{+} & =1-\frac{m}{R}=1-\frac{a^{2} r^{2}}{4(a t+1)^{2}} .
\end{aligned}
$$

Thus,

$$
m=\frac{a^{2} r^{3}}{8(a t+1)^{\frac{3}{2}}} .
$$

To proceed, we calculate the following derivatives:

$$
\begin{aligned}
\ddot{U}= & \frac{\sqrt{a \hat{t}}}{(r+2 \hat{t})^{2}}\left[2 \hat{t} \dot{r}^{2}-(4 \hat{t}+3 r) \dot{t} \dot{r}+2 \hat{t}\left(2 \hat{t} \ddot{t}-2 \hat{t} \ddot{r}+\dot{t}^{2}\right)\right. \\
& \left.+r\left(2 \hat{t} \ddot{t}-2 \hat{t} \ddot{r}+3 \dot{t}^{2}\right)\right] \\
\ddot{R}= & \sqrt{a \hat{t} \ddot{r}}+\frac{2 \sqrt{a} \hat{t} \dot{t}+\sqrt{a}\left(2 \hat{t} \dot{t} \dot{r}+2 \hat{t} r \ddot{t}-r \dot{t}^{2}\right)}{4 \hat{t}^{3 / 2}} \\
\frac{d m(U)}{d U}= & \frac{\dot{m}}{\dot{U}}=\frac{3 r^{2}(r+2 \hat{t})(r \dot{t}-2 \hat{t} \dot{r})}{32 \hat{t}^{4}(\dot{r}-\dot{t})} .
\end{aligned}
$$

where $\hat{t}=t+a^{-1}$. Substitute the above results into Eq. (77) and according to Eq. (74), let the right-hand side of Eq. (76) be equal to the right-hand side of Eq. (77). After a lengthy calculation, we obtain the following result, which is surprisingly simple

$(\dot{t}-\dot{r})(\dot{t}+\dot{r})^{2}=0$

Obviously, the solution is $\dot{r}=\dot{t}$ or $\dot{r}=-\dot{t}$. But this means that the hypersurface is null, inconsistent with our assumption. Therefore, we conclude that the two patches of spacetime cannot be matched through a timelike hypersurface if the continuity of the extrinsic curvature is required. This result is expected because the HMN scalar field is massless and the Vaidya solution describes null dust.

\section{B Self-similarity of HMN spacetime}

In this appendix, we will prove that the HMN spacetime has CSS (continuous self similarity) only when $c=0$ and $a \neq 0$.

A spacetime has CSS if there exists a conformal Killing vector field $\xi^{a}$ satisfying [10]

$\nabla_{(a} \xi_{b)}=g_{a b}$

It is easy to check that Eq. (91) implies $\nabla^{c} \xi_{c}=4$.

Due to spherical symmetry, we can write

$\xi^{a}=x\left(\frac{\partial}{\partial t}\right)^{a}+y\left(\frac{\partial}{\partial r}\right)^{a}$.

Here, $x$ and $y$ are functions of $r$ and $t$. Substituting this expression into Eq. (91), we find that

$$
\begin{aligned}
& y R_{, r}+x R_{, t}=R, \\
& y v_{, r}+x v_{, t}+y_{, r}=1, \\
& y \lambda_{, r}+x \lambda_{, t}+x_{, t}=1, \\
& m y_{, t}-n x_{, r}=0,
\end{aligned}
$$

where

$$
R^{2}=(a t+1) r^{2}\left(1-\frac{2 c}{r}\right)^{1-\alpha},
$$




$$
\begin{aligned}
& \lambda=\frac{1}{2} \log \left[(a t+1)\left(1-\frac{2 c}{r}\right)^{\alpha}\right], \\
& v=\frac{1}{2} \log \left[(a t+1)\left(1-\frac{2 c}{r}\right)^{-\alpha}\right], \\
& m=(a t+1)\left(1-\frac{2 c}{r}\right)^{-\alpha}, \\
& n=(a t+1)\left(1-\frac{2 c}{r}\right)^{\alpha} .
\end{aligned}
$$

From Eq. (93), we can get

$$
x=-\frac{1}{a r(-2 c+r)}(1+a t)\left(4 c r-2 r^{2}-2 c y+\sqrt{3} c y+2 r y\right) .
$$

Substituting Eq. (98) into Eq. (94), we obtain

$y=\sqrt{r} \sqrt{-2 c+r} D(t)$,

where $D(t)$ is an integration function of $t$. Putting Eqs. (98) and (99) into Eq. (96), we find

$$
-\frac{c\left(1-\frac{2 c}{r}\right)^{-\sqrt{3}}((-2+\sqrt{3}) c-\sqrt{3} r)}{a(-2 c+r)^{2} r^{2}}=-\frac{D^{\prime}(t)}{(1+a t) D(t)} \equiv C_{0} .
$$

Obviously, $C_{0}$ must be a constant independent of $r$ and $t$. So the only solution is

$c=0$,

and consequently

$D(t)=D_{0}$.

Now Eqs. (98) and (99) become

$x=-2 a^{-1}(a t+1)\left(D_{0}-1\right)$,

$y=D_{0} r$.

Plugging Eqs. (103) and (104) into Eq. (95), we have

$D_{0}=\frac{2}{3}$

Hence,

$$
\begin{aligned}
x & =\frac{2(1+a t)}{3 a}, \quad y=\frac{2}{3} r \\
\xi^{a} & =\frac{2(1+a t)}{3 a}\left(\frac{\partial}{\partial t}\right)^{a}+\frac{2 r}{3}\left(\frac{\partial}{\partial r}\right)^{a} .
\end{aligned}
$$

Thus, we have proven that the HMN spacetime has continuous self-similarity only for $c=0$ and $a \neq 0$.

\section{References}

1. J.R. Oppenheimer, H. Snyder, On Continued Gravitational Contraction. Phys. Rev. 56, 455 (1939)

2. D. Christodoulou, The structure and uniqueness of generalized solutions of the spherically symmetric Einstein-scalar equations. Commun. Math. Phys. 109, 591 (1987)

3. S. Goncalves, I. Moss, Classical and Quantum Gravity, Black hole formation from massive scalar fields. Class. Quantum Gravit. 14, 2607 (1997)

4. R.G. Cai, L.W. Ji, R.Q. Yang, Collapse of Self-Interacting Scalar Field in Anti-de Sitter Space. Commun. Theor. Phys. 65(03), 329 (2016)

5. S. Chakrabarti, N. Banerjee, Scalar field collapse in a conformally flat spacetime. Eur. Phys. J. C 77, 166 (2017)

6. S. Chakrabarti, Collapsing spherical star in Scalar-Einstein-GaussBonnet gravity with a quadratic coupling. Eur. Phys. J. C 78, 296 (2018)

7. N. Deppe, L.E. Kidder, M.A. Scheel, S.A. Teukolsky, Critical behavior in 3D gravitational collapse of massless scalar fields. Phys. Rev. D 99, 024018 (2019)

8. M. Choptuik, Universality and scaling in gravitational collapse of a massless scalar field. Phys. Rev. Lett. 70, 9 (1993)

9. C. Gundlach, Choptuik spacetime as an eigenvalue problem. Phys. Rev. Lett. 75, 3214 (1995)

10. C. Gundlach, J.M. Martin-Garcia, Critical phenomena in gravitational collapse. Living Rev. Rel. 10, 5 (2007)

11. P.R. Brady, Analytic example of critical behaviour in scalar field collapse. Class. Quant. Grav. 11, 1255 (1994)

12. J. Soda, K. Hirata, Higher dimensional self-similar spherical symmetric scalar field collapse and critical phenomena in black-hole formation. Phys. Lett. B. 387, 271 (1996)

13. A. Wang, H.P. de Oliveira, Critical phenomena of collapsing massless scalar wave packets. Phys. Rev. D 56, 753 (1997)

14. A. Wang, J.F.V. da Rocha, N.O. Santos, Gravitational collapse of a massless scalar field and radiation fluid. Phys. Rev. D 56, 7692 (1997)

15. J. Guo, H. Zhang, Dynamics of critical collapse. Eur. Phys. J. C 79, 625 (2019)

16. J. Celestino, T.W. Baumgarte, Critical collapse of ultrarelativistic fluids: Damping or growth of aspherical deformations. Phys. Rev. D 98, 024053 (2018)

17. B. Kain, Stability and critical behavior of gravitational monopoles. Phys. Rev. D 97, 024012 (2018)

18. C. Gundlach, T.W. Baumgarte, Critical gravitational collapse with angular momentumII. Soft equations of state, Phys. Rev. D 97, 064006 (2018)

19. M. Maliborski, O. Rinne, Critical phenomena in the general spherically symmetric Einstein-Yang-Mills system. Phys. Rev. D 97, 044053 (2018)

20. T. Ikeda, C.M. Yoo, V. Cardoso, Self-gravitating oscillons and new critical behavior. Phys. Rev. D 96, 064047 (2017)

21. J. Jamuna, C. Gundlach, Critical collapse of a rotating scalar field in $2+1$ dimensions. Phys. Rev. D 95, 084001 (2017)

22. T. Ikeda, C.M. Yoo, Critical behavior of a spherically symmetric domain wall collapse. Phys. Rev. D 94, 124032 (2016)

23. G.C. McVittie, The mass-particle in an expanding universe. Mon. Not. R. Astr. Soc. 93, 325 (1933)

24. V. Husain, E.A. Martinez, D. Nunez, Exact solution for scalar field collapse. Phys. Rev. D 50, 3783 (1994)

25. V. Faraoni, S.D. Belknap-Keet, New inhomogeneous universes in scalar-tensor and $f(R)$ gravity. Phys. Rev. D 96, 044040 (2017)

26. D. Kastor, J. Traschen, Building cosmological frozen stars. Class. Quant. Grav. 34, 035012 (2017) 
27. V. Faraoni, Embedding black holes and other inhomogeneities in the universe in various theories of gravity: a short review. Universe 4, 109 (2018)

28. V. Faraoni, Cosmological and black hole apparent horizons. Lect. Notes Phys. 907, 1 (2015)

29. V. Faraoni, D.K. iftci, S.D. Belknap-Keet, Symmetry of BransDicke gravity as a novel solution-generating technique. Phys. Rev. D 97, 064004 (2018)

30. V. Faraoni, V. Vitagliano, T.P. Sotiriou, S. Liberati, Dynamical apparent horizons in inhomogeneous Brans-Dicke universes. Phys. Rev. D 86, 064040 (2012)

31. T. Clifton, D.F. Mota, J.D. Barrow, Inhomogeneous gravity, Mon. Not. R. Astr. Soc. 358, 601 (2005)
32. C. Barrabes, W. Israel, Thin shells in general relativity and cosmology: The lightlike limit. Phys. Rev. D 43, 1129 (1991)

33. W. Israel, Singular hypersurfaces and thin shells in general relativity. Nuovo Cimento B 44, 1 (1966)

34. C.W. Misner, D.H. Sharp, Relativistic equations for adiabatic, spherically symmetric gravitational collapse. Phys. Rev. 136, B571 (1964)

35. F. Fayos, R. Torres, A class of interiors for Vaidya's radiating metric: singularity-free gravitational collapse. Class. Quantum Grav. 25, 175009 (2008) 